\title{
MicroRNA-4516 suppresses pancreatic cancer development via negatively regulating orthodenticle homeobox 1
}

\author{
Shuo Chen ${ }^{1}$, Meng $\mathrm{Xu}^{1}{ }^{*}$, Jing Zhao ${ }^{2}$, Jiaqi Shen ${ }^{3}$, Junhui Li ${ }^{1}$, Yang Liu ${ }^{1}$, Gang Cao ${ }^{1}$, Jiancang Ma ${ }^{1}$, Weizhou \\ $\mathrm{He}^{4}, \mathrm{Xi}_{\mathrm{C}} \mathrm{Chen}^{1}{ }^{\otimes}, \mathrm{Tao} \mathrm{Shan}^{1}{ }^{\bowtie}$ \\ 1. Department of General Surgery, The Second Affiliated Hospital of Xi'an Jiaotong University, Xi'an Jiaotong University, PR China \\ 2. School of Science, Xi'an Jiaotong University, PR China \\ 3. School of Life Science, Xiamen University, PR China \\ 4. Department of Hepatobiliary Surgery, The First Affiliated Hospital of Xi'an Jiaotong University, Xi'an Jiaotong University, PR China \\ *These authors contribute equally. \\ \ Corresponding author: Xi Chen; E-mail: chenxijiaoda@163.com. Tao Shan; E-mail: shantao820304@163.com
}

() The author(s). This is an open access article distributed under the terms of the Creative Commons Attribution License (https://creativecommons.org/licenses/by/4.0/). See http://ivyspring.com/terms for full terms and conditions.

Received: 2020.03.12; Accepted: 2020.04.20; Published: 2020.05.18

\begin{abstract}
Pancreatic cancer remains one of the most lethal human cancers without efficient therapeutic strategy. MicoRNAs (miRNAs) are a group of small non-coding RNAs involved in multiple biological processes including tumor development and progression. In this study, we investigated the expression and function of miR-4516 in pancreatic cancer. MiR-4516 was low-expressed in pancreatic cancer tissues and cell lines. Overexpression of miR-4516 inhibited pancreatic cancer cell proliferation, migration and invasion, while promoted cell apoptosis in vitro. Further, overexpression of miR-4516 suppressed xenograft pancreatic tumor growth in vivo. Bioinformatics analysis was performed and miR-4516 was predicted to negatively regulate orthodenticle homeobox 1 (OTX1) expression by binding to its 3'-UTR. Consistently, OTX1 was highly expressed in pancreatic cancer tissues and cell lines. Knockdown of OTX1 expression suppressed pancreatic cancer cell migration and invasion, with down-regulated MMP2 and MMP9 expression. Moreover, we demonstrated that miR-4516 regulated pancreatic cancer cell growth, migration, invasion and apoptosis via targeting OTX1. Overexpression of OTX1 could partially abrogate the inhibitory effect of miR-4516. Taken together, we conclude that miR-4516 could function as a tumor suppressor via targeting OTX1. These findings suggest that miR-4516/OTX1 axis might be a novel therapeutic target for miRNA-based therapy for pancreatic cancer patients.
\end{abstract}

Key words: miR-4516; pancreatic cancer; OTX1; miRNA-based therapy

\section{Introduction}

Pancreatic cancer is one of the most lethal cancers and ranks $7^{\text {th }}$ leading cause of global cancer deaths in developed countries [1]. Though great advances have been achieved in surgical technique, chemotherapy and radiotherapy, the therapeutic outcomes remain poor due to the late diagnosis [2-4]. The 5-year relative survivals of pancreatic cancer were only $7.2 \%$ in China and the lowest level in all cancers [5]. Also, the definite cure strategy is still not available to treat the pancreatic patients with advanced-stages $[6,7]$. Thus, it is critical to identify the novel diagnostic markers to screen for the patients at early stage and develop new efficient therapeutic strategies to treat late stage pancreatic cancer patients.

MicroRNAs (miRNAs) are a subfamily of non-coding RNAs which post-transcriptionally regulate gene expression and function in various biological process including cancer development $[8$, 9]. MiRNAs play an important role in tumor development and progression, acting as tumor suppressor or oncogene [10]. Mounting evidence has demonstrated that multiple miRNAs are involved in 
pancreatic cancer tumorigenesis and metastasis. Taurine-upregulated gene 1 (TUG1) was reported as miR-299-3p sponge and inhibition of TUG1/miR-299-3p expression dampened pancreatic cancer progression via suppressing the Notch1 signaling pathway [11]. Hypoxia-induced miR-646 impaired the stability of Migration and invasion inhibitory protein (MIIP) mRNA and inhibition of miR-646 repressed proliferation and invasion capability of pancreatic cancer cells [12]. In contrast, miR-399-5p inhibited the migration and invasion of pancreatic cancer cells via targeting ZNF689 [13]. Jiang $W$. et al. reported that miR-101 suppressed epithelial-to-mesenchymal transition via targeting HMGA2 in pancreatic cancer cells. MiR-4516 is a newly identified miRNA involved in autophagy associated with exposure to fine particulate matter [14]. A recent study demonstrated that miR-4516 might function as an oncogene in human glioblastoma via targeting PTPN14 [15]. However, the expression and function of miR-4516 in pancreatic cancer remains largely unknown.

Orthodenticle homeobox 1 (OTX1) is a transcription factor regulating brain and sensory organ development $[16,17]$. OTX1 was demonstrated to be highly expressed in colorectal cancer and breast cancer as an oncogene [18, 19]. Downregulation of OTX1 inhibited cell proliferation, migration and invasion in gastric cancer [20]. The role of OTX1 in pancreatic cancer is not clear.

In this study, we demonstrated that miR-4516 was downregulated in pancreatic cancer and cell lines while OTX1 was highly expressed in pancreatic cancer and cell lines. MiR-4516 negatively regulated OTX1 expression by binding to its $3^{\prime}$-UTR. Overexpression of miR-4516 suppressed pancreatic cancer cell proliferation, migration and invasion, while promoted cell apoptosis via regulating OTX1. Together, we demonstrate that miR-4516/OTX1 axis might be a novel therapeutic target for pancreatic cancer treatment.

\section{Materials and Methods}

\section{Patient Specimens}

Paired pancreatic cancer tissues and adjacent noncancerous tissues were obtained from pancreatic patients undergoing surgery in The Second Affiliated Hospital of Xi'an Jiaotong University. Samples were snap-frozen and stored at $-80^{\circ} \mathrm{C}$ until further use. Informed consent was signed by all patients. The study was approved by the Research Ethics Committee of The Second Affiliated Hospital of Xi'an Jiaotong University.

\section{Cell culture}

Human pancreatic cancer cell line SW1990, CAPAN-1, PANC-1 and AsPC-1, and control cell line HPDE6-C7 were obtained from American Type Culture Collection (ATCC, Manassas, VA, USA). All the cell lines were cultured with RPMI1640 medium containing 10\% fetal bovine serum (Gibco), $100 \mathrm{U} / \mathrm{ml}$ penicillin, $100 \mu \mathrm{g} / \mathrm{ml}$ streptomycin in a cell incubator with $5 \% \mathrm{CO}_{2}$ at $37^{\circ} \mathrm{C}$.

\section{Transfection}

Cell transfection was performed using lipofectamine 3000 (Invitrogen) following the manufacturer's protocol. The miR-NC, miR-4516 mimics, miR-4516 inhibitor and inhibitor negative control were purchased from Ribobio (Guangzhou, China). OTX1 overexpression vector was constructed by cloning the OTX1 ORF into pcDNA3.1 vector.

\section{RT-qPCR}

Total RNA was purified from tissues or cell lines using Trizol (Invitrogen) and reverse-transcribed into complementary DNA using a reverse-transcription kit (Applied Biosystems). The expression of miR-4516 and potential target genes were measured by quantitative PCR (qPCR) using SYBR Green PCR Kit (Applied Biosystems) on an Applied Biosystems Real-time PCR machine. The relative expression was normalized to internal control human U6 RNA or GAPDH RNA using 2- $\triangle \triangle \mathrm{CT}$ methods. The primers used in the study were listed below: for miR-4516, 5'-ATGGGAGAAGGGTCGGGG-3'; for OTX1， 5' -CTGCTCTTCCTCAATCAATGG-3' (forward) and 5' -ACCCTGACTTGTCTGTTTCC-3' (reverse); for GAPDH, 5'-CCATCACCATCTTCCAGGAG T-3' (forward) and 5'-GGATGATGTTCTGGAGAGCG-3' (reverse).

\section{Fluorescence In Situ Hybridization}

The expression of miR-4516 in pancreatic tissues and control noncancerous tissues were examined by Fluorescence In Situ Hybridization as described before[21]. Paraffin-embedded 4\%-PFA-fixed tissues were cut into $6 \mu \mathrm{m}$ sections and deparaffinized. The antigen was retrieved by boiling in citric acid buffer in a water bath for $20 \mathrm{~min}$. Proteinase K $(200 \mu \mathrm{L}$; Servicebio, Wuhan, China) in PBS was added to the sections in a humidified chamber, which were then incubated for $25 \mathrm{~min}$ at $37^{\circ} \mathrm{C}$ and washed twice with PBS for 5 min each. Prehybridization buffer $(100 \mu \mathrm{L}$; Servicebio) was added to each tissue section. The sections were placed in a hybridization chamber, incubated for $1 \mathrm{~h}$ at $37^{\circ} \mathrm{C}$. The prehybridization buffer was replaced with hybridization buffer containing the FAM-labeled miR-4516 probe. The samples were 
allowed to hybridize overnight at $37^{\circ} \mathrm{C}$. DAPI (Servicebio) was used for nuclear staining.

\section{CCK-8 assay}

Cells were seeded into 96-well plates (2000 cells/well) and incubated for indicated time after transfection. A Cell counting Kit-8 (CCK-8, dojindo) kit was used to evaluate the cell viability at indicated time points. The assay was repeated independently for 3 times.

\section{EdU staining assay}

5-ethynyl-20-deoxyuridine (EdU) assay was performed to assess the DNAY synthesis rate using EdU assay Kit (Ribobio, Guangzhou, China). The staining was recorded and analyzed with a microscopy. The ratio of EdU positive cells (Green) to DAPI-stained cells (Blue) was analyzed to calculate the cell proliferation rate.

\section{Cell apoptosis assay}

Cells were collected 72 hours post transfection and stained with FITC-Annexin V/Propidium Iodide (Sigma). Cell apoptosis was analyzed using flow cytometry and Annexin V+PI- cells were defined as apoptotic cells.

\section{Transwell assay}

Cell invasion and migration was evaluated using Transwell chambers with or without precoated Matrigel layer. Briefly, 30000 cells were seeded into the upper chamber in serum-free medium, the lower chamber was added with medium containing 30\% FBS. After incubation for 48 hours, the migrated or invaded cells were fixed with $4 \%$ paraformaldehyde and stained with $0.1 \%$ crystal violet. The migrated and invaded cells were counted under a light microscopy.

\section{Luciferase reporter assay}

PANC-1 or AsPC-1 cells were seeded into 96-well plates (4000 cells/well) and transfected with luciferase reporter vector containing WT or mutated 3'-UTR of OTX1 (Constructed by Ribobio Company), together with miR-4516 or miR-NC. After 48 hours, the relative luciferase activity was analyzed using the Dual-luciferase reporter assay system (Promega) following the manual.

\section{Western Blot}

Total protein was prepared from tissues or cultured cells using RIPA buffer (Beyotime, China). Equal amount of protein was separated by SDS-PAGE and transferred to polyvinylidene difluoride memebranes (Millipore). The membrane was incubated with primary antibodies against OTX1
(Abcam, ab25985), $\beta$-actin (Abcam, ab8227) and then incubated with HPR conjugated secondary antibody. Protein bands were visualized using chemiluminescence reagents (ECL, Pierce).

\section{Immunohistochemical staining}

The immunohistochemical staining of paraffin-embedded tissues was conducted as previously described[22]. Tissue samples was stained with primary antibody anti-OTX1 (Abcam, ab25985) and further incubated with biotinylated goat anti-rabbit secondary antibody (Cell signaling, \#14708). Then 3, 3-diaminobenzidine was used to visualize the OTX1 expression.

\section{Xenograft tumor model}

BALB/C nude mice (5-week old) were obtained from Shanghai SLAC Animal Center (Shanghai, China). PANC-1 cells stably overexpression of miR-4516 or control PANC-1 cells $\left(6 \times 10^{6}\right)$ were inoculated subcutaneously into the right flanks of mice. Tumor growth was monitored every 4 days and tumor volume was calculated as volume $(\mathrm{mm} 3)=$ length $\times$ width $^{2} / 2$. Mice were euthanized at day 17 and the tumor weights were assessed. The animal experiments were approved by the Animal Care and Use Committee of the Second Affiliated Hospital of Xi'an Jiaotong University.

\section{Statistics analysis}

All data were analyzed using GraphPad Prism V6 and expressed as mean \pm SD. Differences between groups was analyzed using the student $t$ test (two groups) or One-way ANOVA (multiple groups) where necessary. A P value $<0.05$ was considered statistically significant.

\section{Results}

\section{MiR-45 16 is low-expressed in pancreatic cancer tissues and cell lines}

To investigate the expression profile of miR-4516 in pancreatic cancer, we first examined the expression of miR-4516 in 10 paired pancreatic cancer tissues and adjacent noncancerous tissues by qPCR. MiR-4516 is significantly low-expressed in pancreatic cancer tissues compared with that in adjacent noncancerous tissues (Figure 1A). In addition, the expression of miR-4516 was much markedly down-regulated in four pancreatic cancer cell lines (SW1990, CAPAN-1, PANC-1 and AsPC-1) in comparison with that in HPDE6-C7 control cell line (Figure 1B). We further confirmed the decreased expression of miR-4516 in pancreatic cancer tissues by in situ hybridization (Figure 1C). Pancreatic cancer cell lines PANC-1 and 
AsPC-1, with the lowest expression of miR-4516, were used for the subsequent experiments.

\section{Overexpression of $\mathrm{miR}-4516$ inhibits} pancreatic cell growth, migration and invasion and promotes cell apoptosis in pancreatic cells

To explore the function of miR-4516 in pancreatic cancer, we transfected PANC-1 and AsPC-1 cells with miR-4516 mimics to overexpression miR-4516. As shown in Figure 2A-2B, CCK-8 cell proliferation assay and EdU staining assay demonstrated that overexpression of miR-4516 significantly repressed cell growth and DNA synthesis. In contrast, cell apoptosis was remarkably enhanced in PANC-1 and AsPC-1 cells transfected with miR-4516 mimics (Figure 2C). Furthermore, cell migration and invasion capability of PANC-1 and AsPC-1 cells transfected with miR-4516 mimics were notably weakened compared with that in cells transfected with negative control miR-NC (Figure 2D).

\section{Overexpression of $\mathrm{miR}-4516$ inhibits pancreatic xenograft tumor growth in vivo}

To gain further insight of the function of miR-4516 in pancreatic cancer, we established a pancreatic cancer xenograft tumor model. PANC-1 cells stably transfected with miR-4516 mimics or miR-NC were transplanted into the nude mice. Overexpression of miR-4516 resulted in suppressed tumor growth (Figure 3A). The xenograft tumors from miR-4516 overexpression groups showed smaller tumor size and lower tumor weight than that from control group (Figure 3B-3C). Consistently, we confirmed that miR-4516 was highly expressed in xenograft tumors from miR-4516 overexpression group (Figure 3D).

\section{MiR-45 16 negatively regulates OTX1 expression by binding to its 3'-UTR}

To understand the functional mechanism of miR-4516 in pancreatic cancer, we performed bioinformatics analysis using different online database to predict the potential targets of miR-4516. As shown in Figure $\mathbf{4 A}$, combined analysis from TargetScan, miRDB and TarBase indicated 13 shared common targets. PANC-1 cells were transfected with miR-4516 mimics or miR-NC to verify the expression levels of these 13 targets. Overexpression of miR-4516 significantly inhibited the expression of OTX1 (Figure 4B). Further analysis revealed that miR-4516 had the
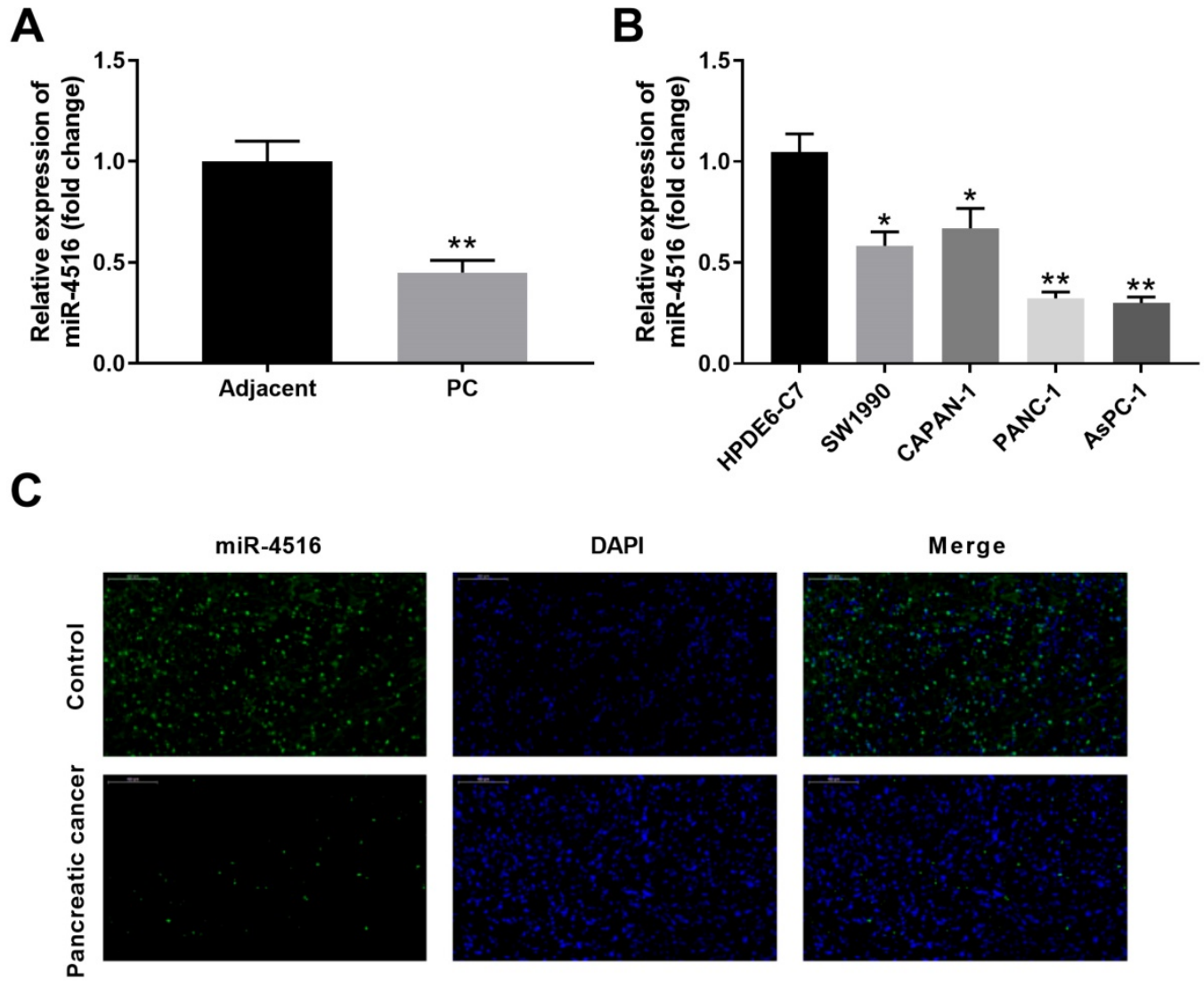

Figure 1. MiR-4516 is low-expressed in pancreatic cancer tissues and cell lines. (A) The expression of miR-4516 in 10 paired pancreatic cancer tissues and adjacent noncancerous tissues was examined by qPCR. (B) The expression of miR-4516 in pancreatic cancer cell lines (SW1990, CAPAN-1, PANC-1 and AsPC-1) and control cell line HPDE6-C7 was examined by qPCR. (C) The expression of miR-4516 in paired pancreatic cancer tissues and adjacent noncancerous tissues was examined by in situ hybridization. $*, P<0.05 ; * *, P<0.01$. 
complementary binding sequences targeting $3^{\prime}$-UTR of OTX1 (Figure 4C). Luciferase reporter vector containing wild-type (WT) or mutated 3'-UTR of
OTX1 was constructed and transfected into PANC-1 or AsPC-1 cells together with miR-4516 mimics or miR-NC.
A

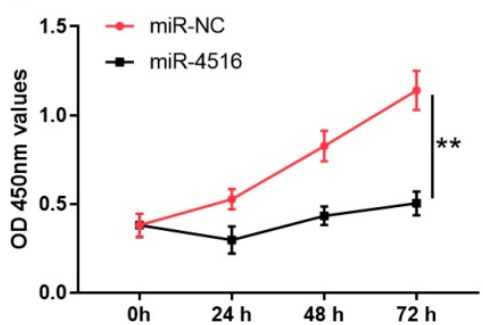

AsPC-1

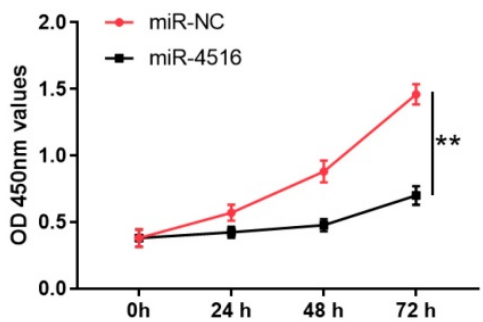

B
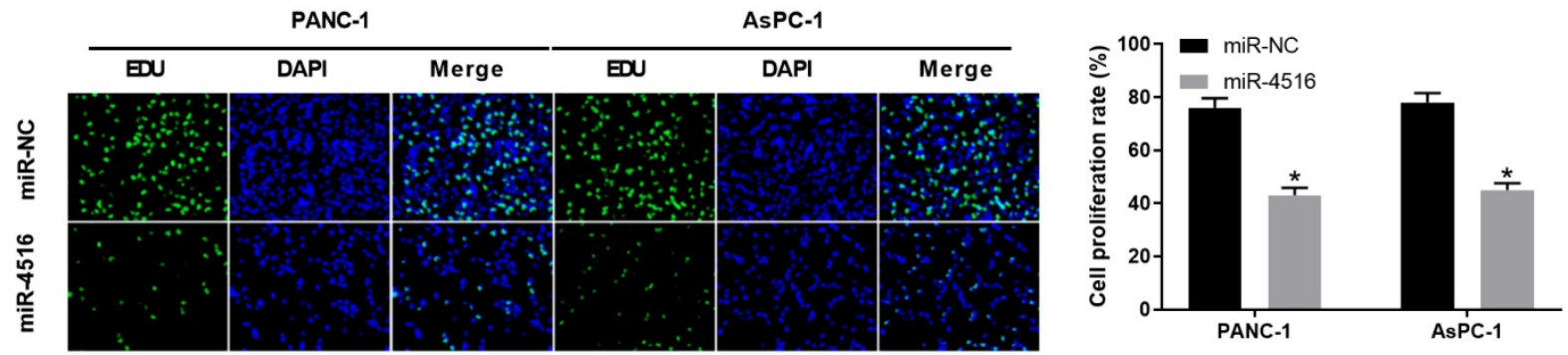

C

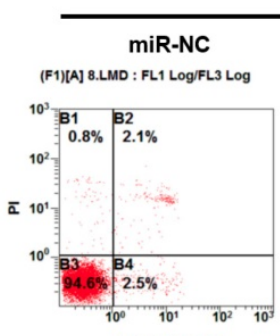

PANC-1

AsPC-1
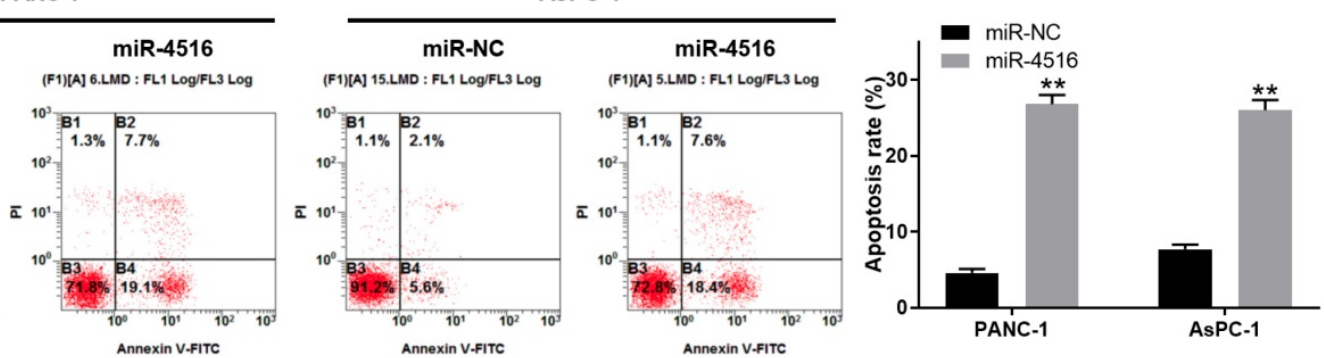

D
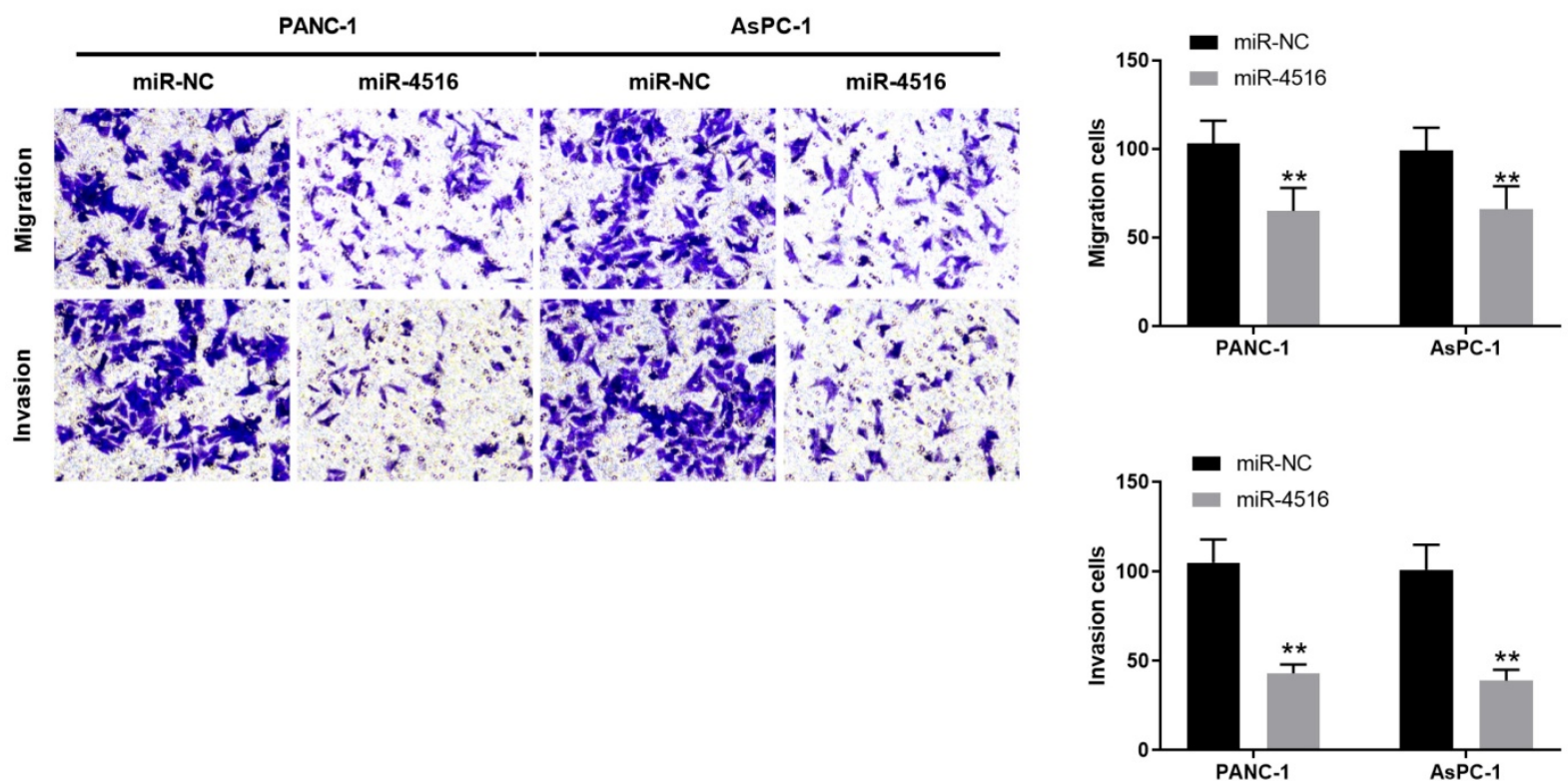

Figure 2. Overexpression of miR-4516 inhibits pancreatic cell growth, migration and invasion and promotes cell apoptosis in pancreatic cells. PANC-1 or AsPC-1 cells were transfected with miR-4516 mimics or negative control miR-NC. (A) Cell viability was analyzed by CCK-8 assay at indicated time points. (B) DNA synthesis was analyzed by EdU/DAPI double staining. (C) Cell apoptosis was analyzed by Annexin V/PI double staining. (D) Cell migration and invasion was analyzed by transwell assay. *, $P<$ $0.05 ; * *, P<0.01$ 


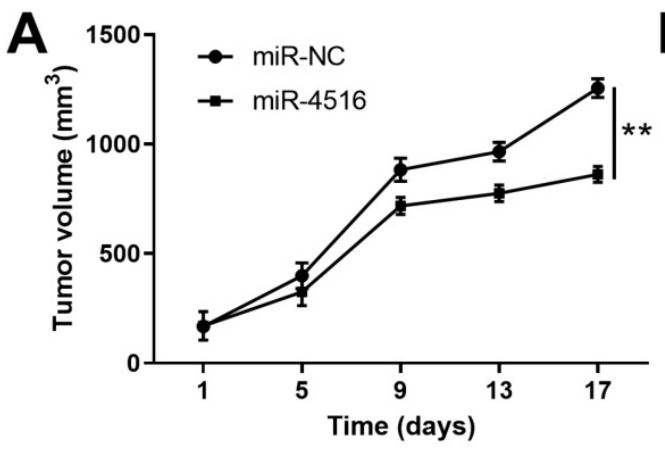

B
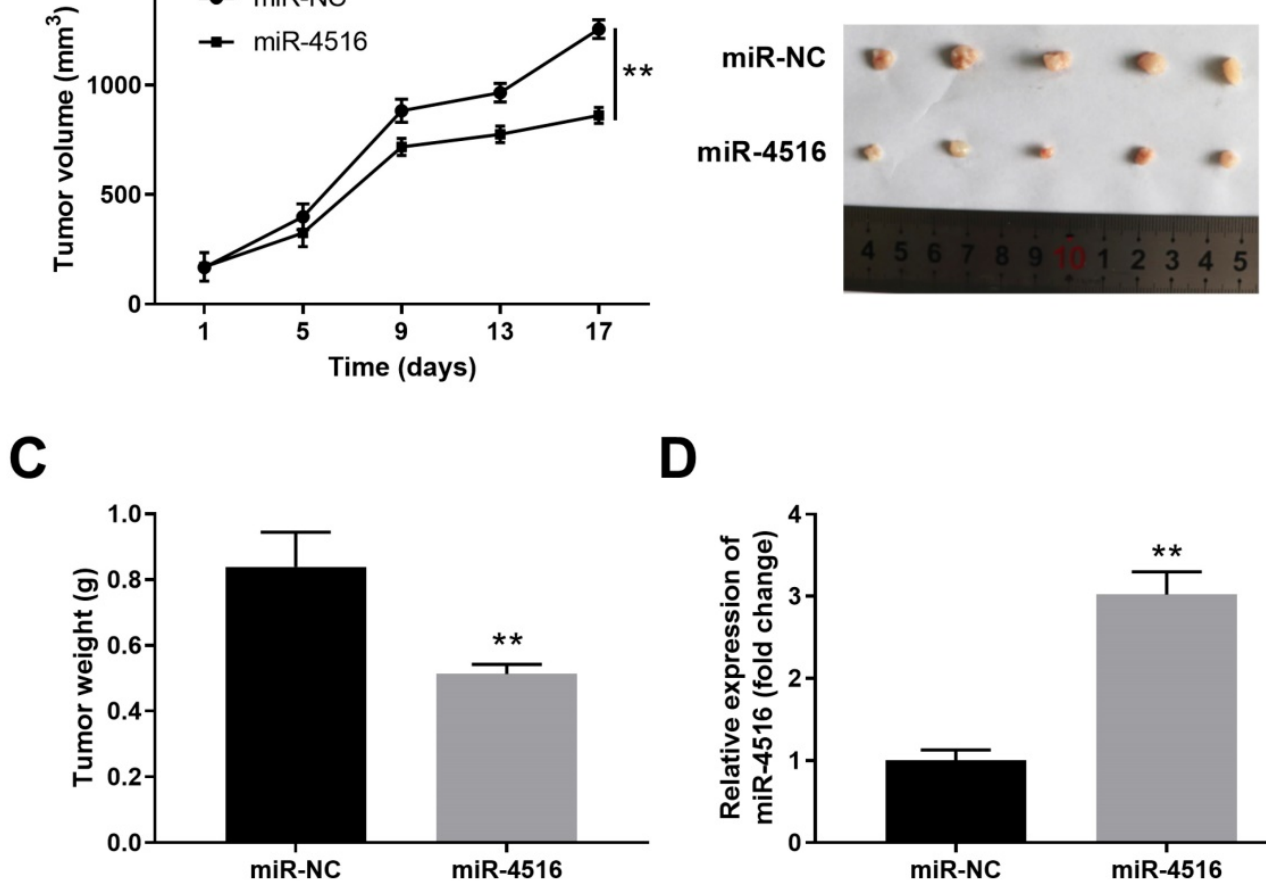

D

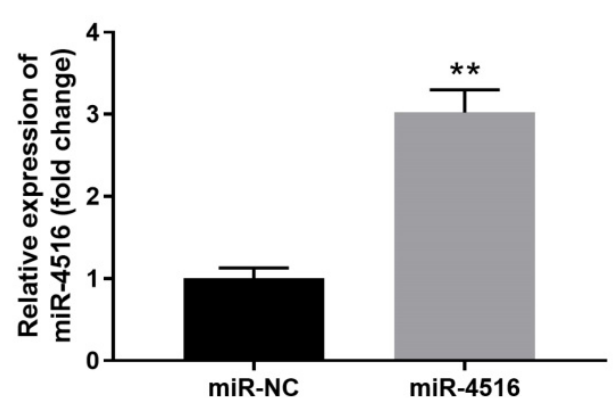

Figure 3. MiR-4516 overexpression inhibits pancreatic xenograft tumor growth in vivo. PANC-1 cells were stably transfected with miR-4516 or negative control miR-NC and implanted into the right flank of BALB/C nude mice. (A) Tumor growth was monitored and the tumor volume was assessed at indicated time points. (B, C) Xenograft tumor from miR-4516 overexpression group and control group was extracted from mice. (B) Tumor size and (C) Tumor weight were analyzed. (D) The expression of miR-4516 was analyzed in xenograft tumors by qPCR. $* *, P<0.01$.

As expected, overexpression of miR-4516 specifically inhibited the luciferase activity in cells transfected with reporter vector containing WT 3'-UTR of OTX1, but not with reporter vector containing mutated $3^{\prime}$-UTR of OTX1 (Figure 4D). In addition, overexpression of miR-4516 inhibited OTX1 expression while inhibition of miR-4516 enhanced OTX1 expression in AsPC-1 cells at both mRNA and protein levels (Figure 4E-4F). Moreover, we found that OTX1 was highly expressed in pancreatic tumor tissues, as demonstrated by immunohistochemical staining (Figure 4G). Pancreatic tumor tissues also had notably lower levels of OTX1 mRNA compared with that in adjacent non-tumor tissues (Figure $\mathbf{4 H}$ ). These findings indicated that miR-4516 might negatively regulate OTX1 by binding to its $3^{\prime}$-UTR.

\section{OTX 1 is highly expressed in pancreatic cancer tissues and knockdown of OTX 1 suppresses pancreatic cell migration and invasion}

We further explored the expression pattern and function of OTX1 in pancreatic cancer. As shown in Figure 5A, OTX1 was highly expressed in pancreatic cancer tissues compared with that in non-tumor tissues. Consistently, pancreatic cancer cell lines had significantly higher level of OTX1 than that in control cell line HPDE6-C7 (Figure 5B). RNA interfence was conducted and siRNA-targeting OTX1 notably repressed the mRNA and protein levels of OTX1 in
PANC-1 and AsPC-1 cells (Figure 5C-5D). Transwell assay demonstrated that knockdown of OTX1 dampened cell migration and invasion in PANC-1 and AsPC-1 cells (Figure 5E-5F). Furthermore, OTX1 knockdown markedly inhibited the expression of MMP2 and MMP9 in PANC-1 and AsPC-1 cells, indicating that OTX1 played a critical role in pancreatic cell migration and invasion (Figure 5G-5H).

\section{MiR-45 16 inhibits pancreatic cell growth, migration and invasion and promotes cell apoptosis via targeting OTX1}

To further verify the regulatory axis of miR-4516/OTX1, we transfected PANC-1 or AsPC-1 cells with miR-NC, miR-4516 mimics, or miR-4516 mimics + OTX1 overexpression vector. As shown in Figure 6A, miR-4516 mimics inhibited the expression of OTX1 while overexpression of OTX1 rescued the OTX1 expression in pancreatic cell lines. Overexpression miR-4516 repressed cell proliferation and DNA synthesis (Figure 6B-6D). However, overexpression of OTX1 partially reversed the inhibitory effect of miR-4516 (Figure 6B-6D). In addition, miR-4516 mimics promoted cell apoptosis while overexpression of OTX1 antagonized the promotion effect of miR-4516 mimics (Figure 6E-6F). Furthermore, transwell assay demonstrated that overexpression of OTX1 abrogated the inhibitory 
effect of miR-4516 mimics in pancreatic cell migration and invasion (Figure 6G-6I). Taken together,
miR-4516 regulated pancreatic cell growth, migration, invasion, and apoptosis via targeting OTX1.
A

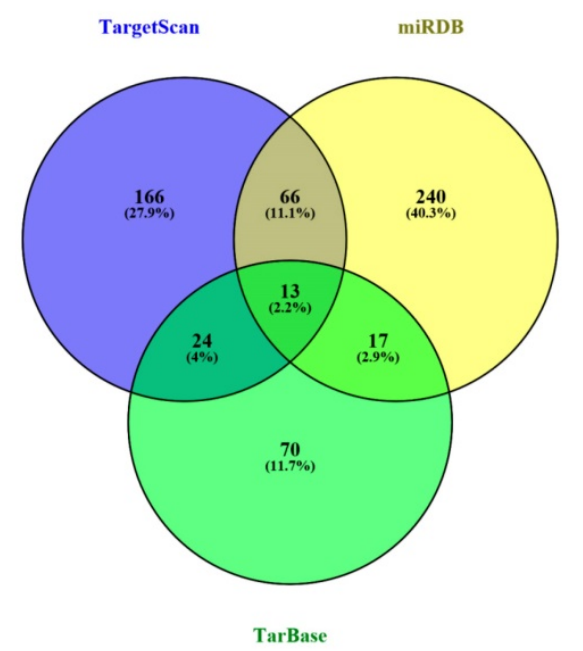

C

WT OTX13'UTR 5' CUGCCCUCUUCACAUCUUCUCCC 3'

$\operatorname{miR}-4516 \quad 3^{\prime}$

CGGGGCUGGGAAGAGGG 5'

MUT OTX1 3'UTR 5' CUGCCCUCUUCACAUCAAGAGCC 3'

B
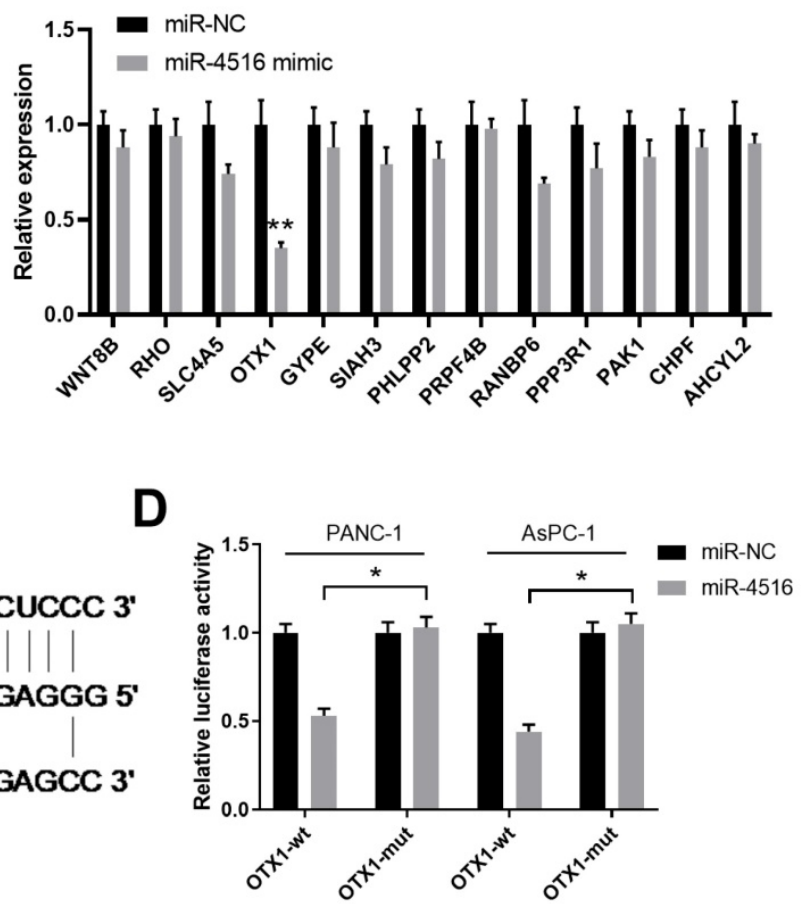

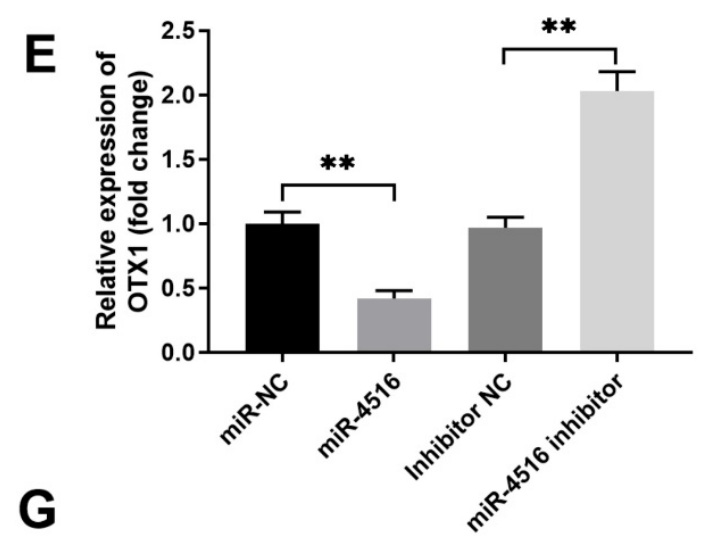

Non-tumor

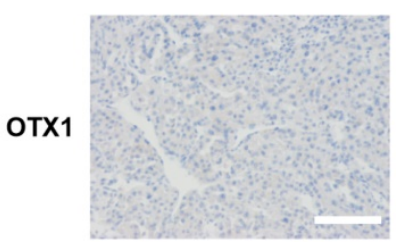

Tumor

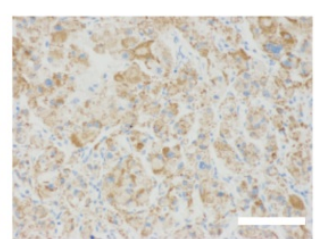

$\mathbf{F}$
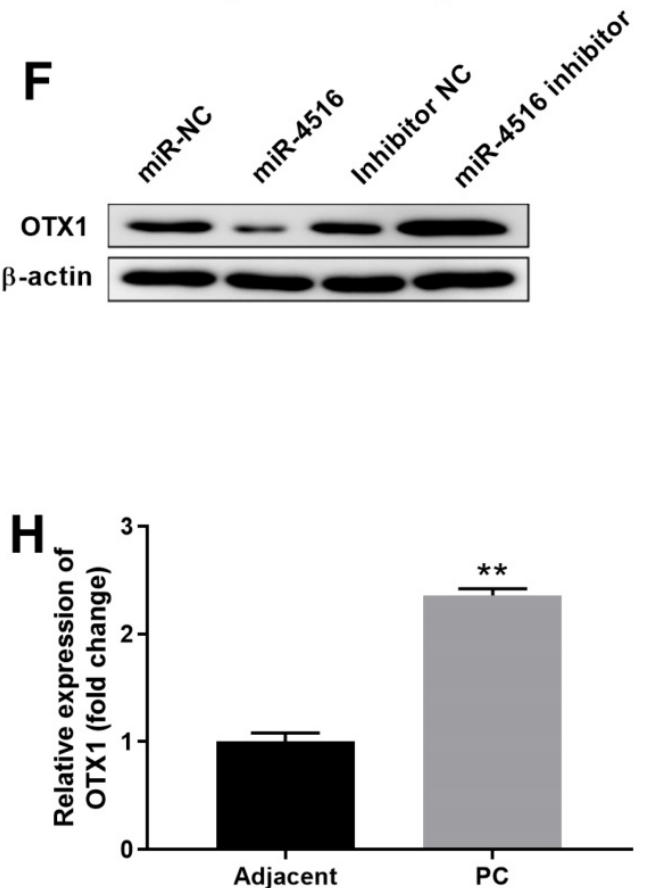

Figure 4. MiR-4516 negatively regulates OTX1 expression by binding to its 3'-UTR. (A) Bioinformatics analysis was performed to predict the potential targets of miR-4516 using online database TargetScan, miRDB, and TarBase. (B) PANC-1 cells were transfected with miR-4516 or miR-NC. Potential target gene expression levels were analyzed by qPCR 48 hours later. (C) The putative binding sequences between miR-4516 and WT 3'-UTR of OTX1 was shown. (D) PANC-1 or AsPC-1 cells were transfected with luciferase reporter vector containing WT or mutated 3'-UTR of OTX1, together with miR-4516 or miR-NC. The relative luciferase activity was analyzed 48 hours later. (E, F) AsPC-1 cells were transfected with miR-NC, miR-4516 mimics, miR-4516 inhibitor or inhibitor negative control. 48 hours later, the mRNA expression (E) and protein expression (F) of OTX1 were analyzed by qPCR and western blot. (G) The expression of OTX1 in pancreatic cancer tissues and adjacent non-tumor tissues was analyzed by immunohistochemical staining. $(\mathrm{H})$ The relative expression of $\mathrm{OTX} 1$ in pancreatic cancer tissues and adjacent non-tumor tissues was analyzed by qPCR. *, $P<0.05$; $* *, P<0.01$. 


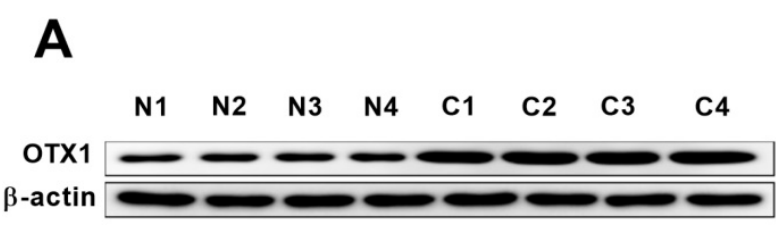

B
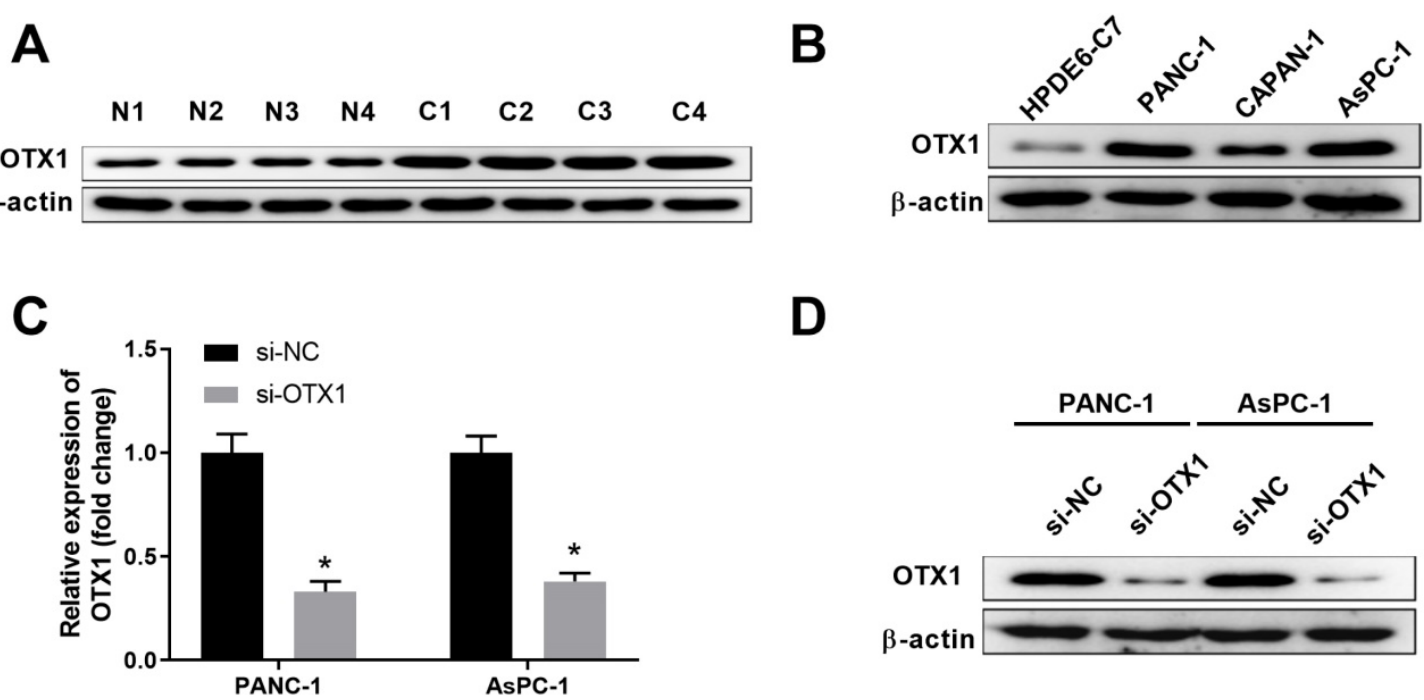

D

$\mathbf{E}$
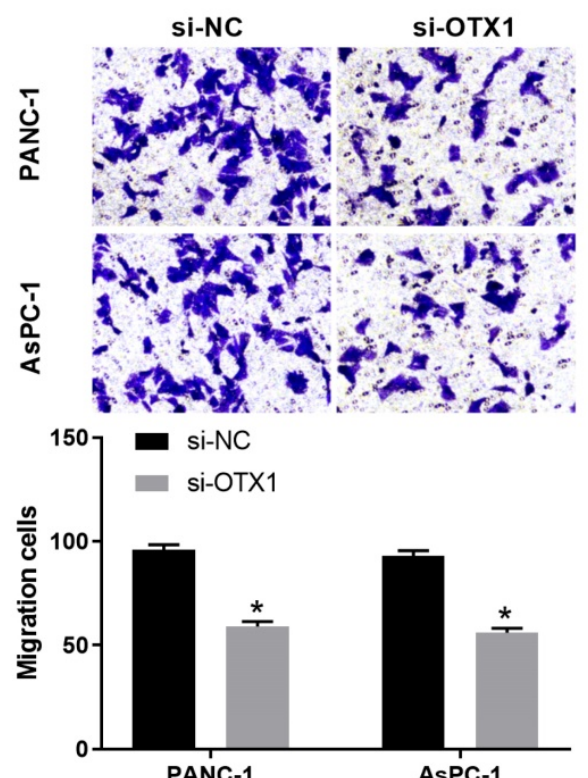

$\mathbf{G}$

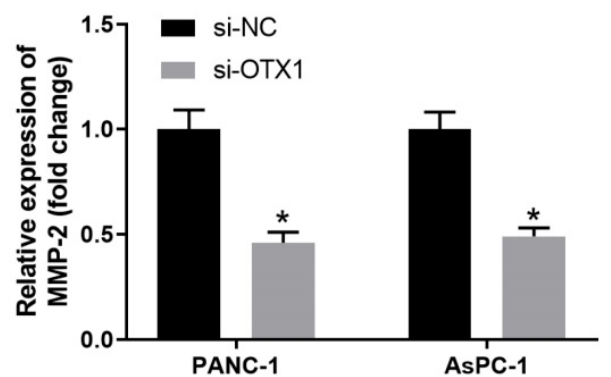

PANC-1 AsPC-1

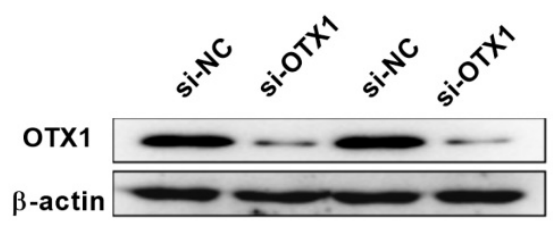

F

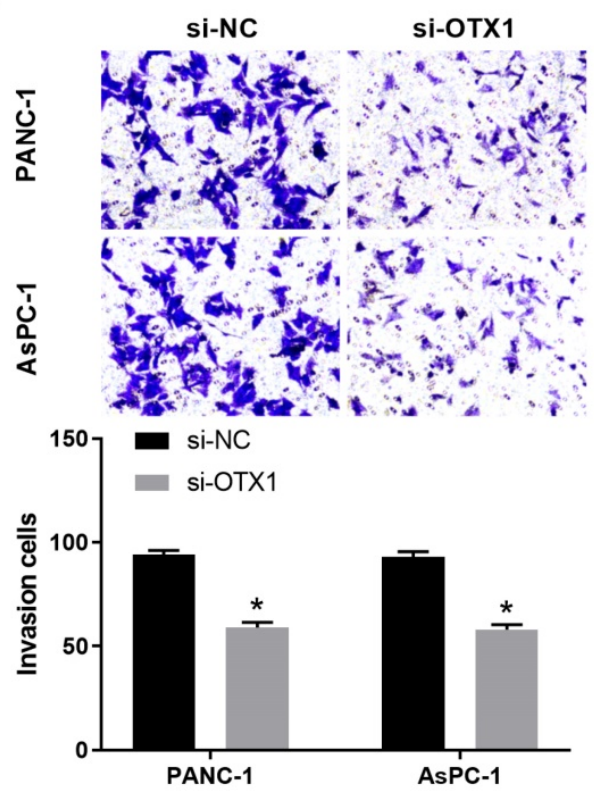

H

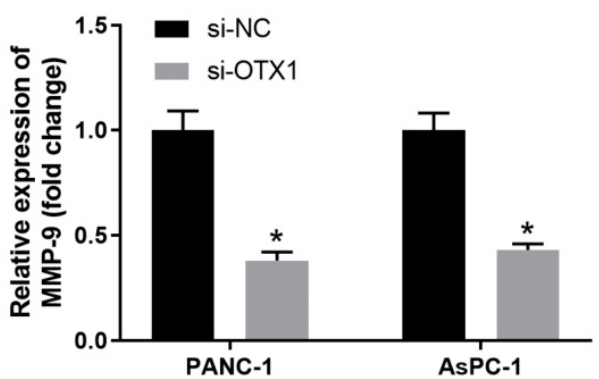

Figure 5. OTX1 is highly expressed in pancreatic cancer tissues and knockdown of OTX1 suppresses pancreatic cell migration and invasion. (A) The protein expression of OTX1 in pancreatic cancer tissues (C) or adjacent non-tumor tissues (N) was analyzed by western blot. (B) The protein expression of OTX1 in pancreatic cancer cell lines (PANC-1, CAPAN-1, and AsPC-1) and control cell line HPDE6-C7 was analyzed by western blot. (C, D) PANC-1 or AsPC-1 cells were transfected with si-NC or si-OTX1. The relative expression of OTX1 mRNA or the protein expression of OTX1 was analyzed by qPCR and western blot 48 hours later. (E, F) Transwell assay was performed to evaluate the migration and invasion capability of PANC-1 or AsPC-1 cells transfected with si-NC or si-OTX1. (G, H) PANC-1 or AsPC-1 cells were transfected with si-NC or si-OTX1. The relative expression of MMP2 and MMP9 was analyzed by qPCR 48 hours later. $*, P<0.05$ 
A

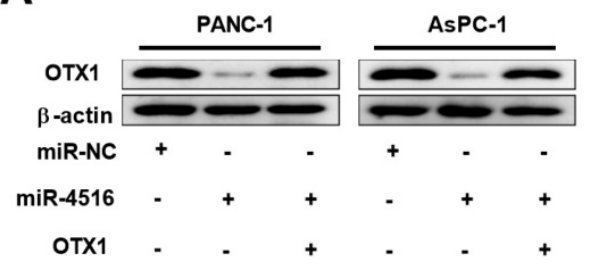

C

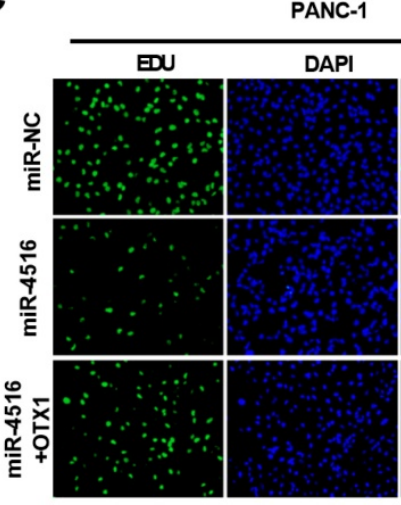

E

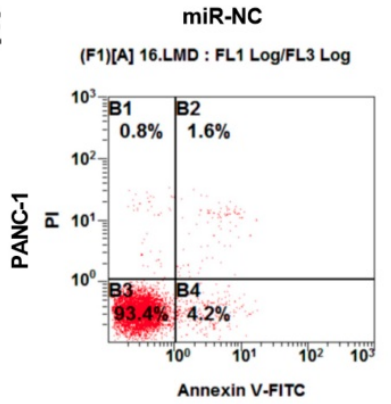

(F1)[A] 23.LMD : FL1 Log/FL3 Log

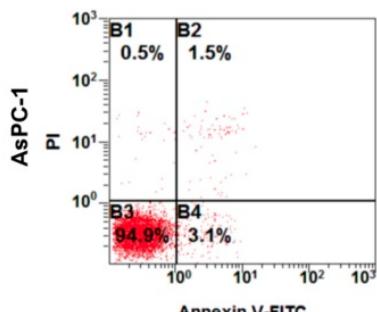

G

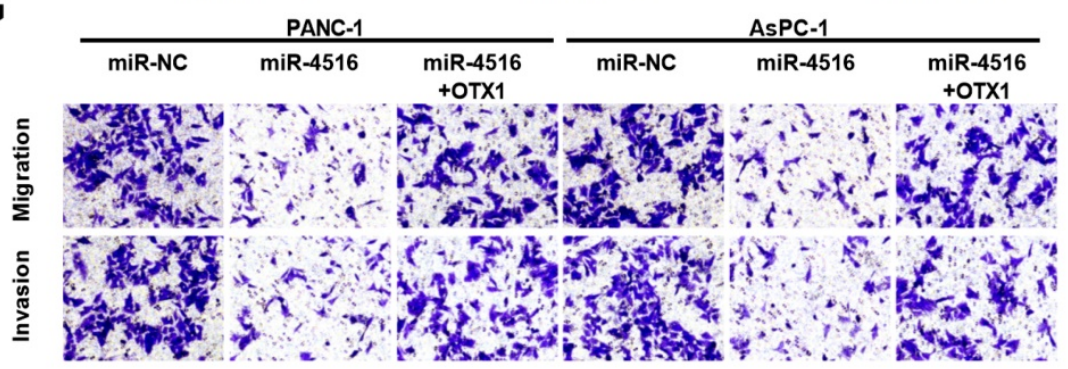

B

AsPC-1
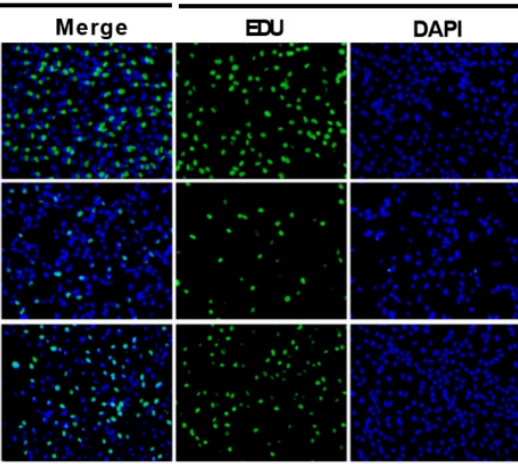

(F1)[A] 4.LMD : FL1 Log/FL3 Log

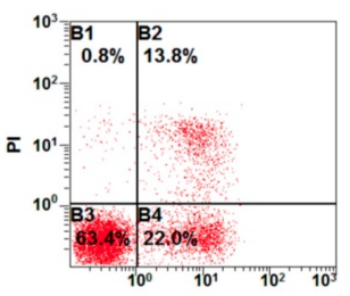

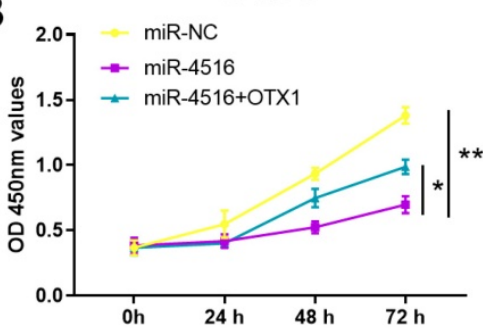
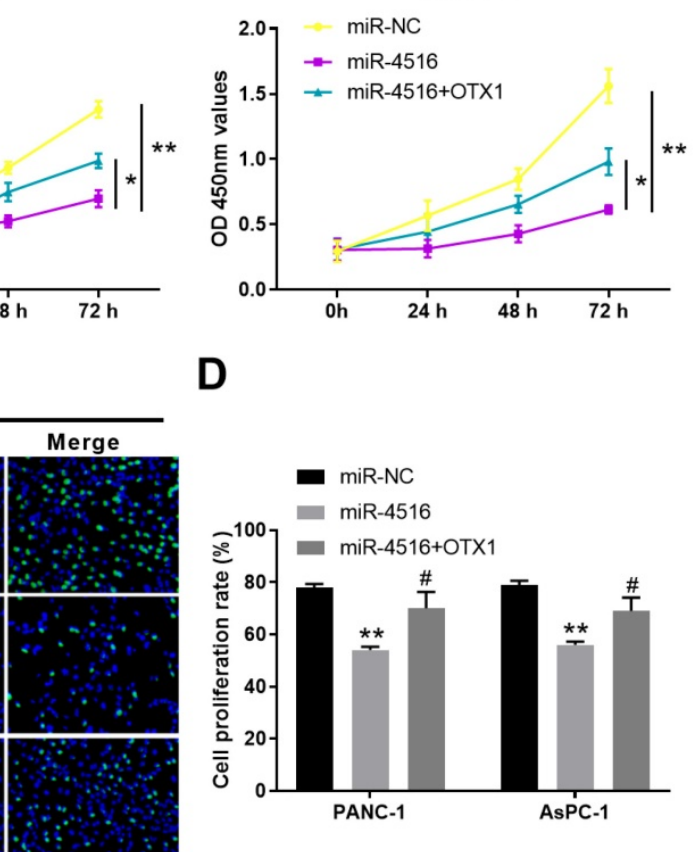

D

F
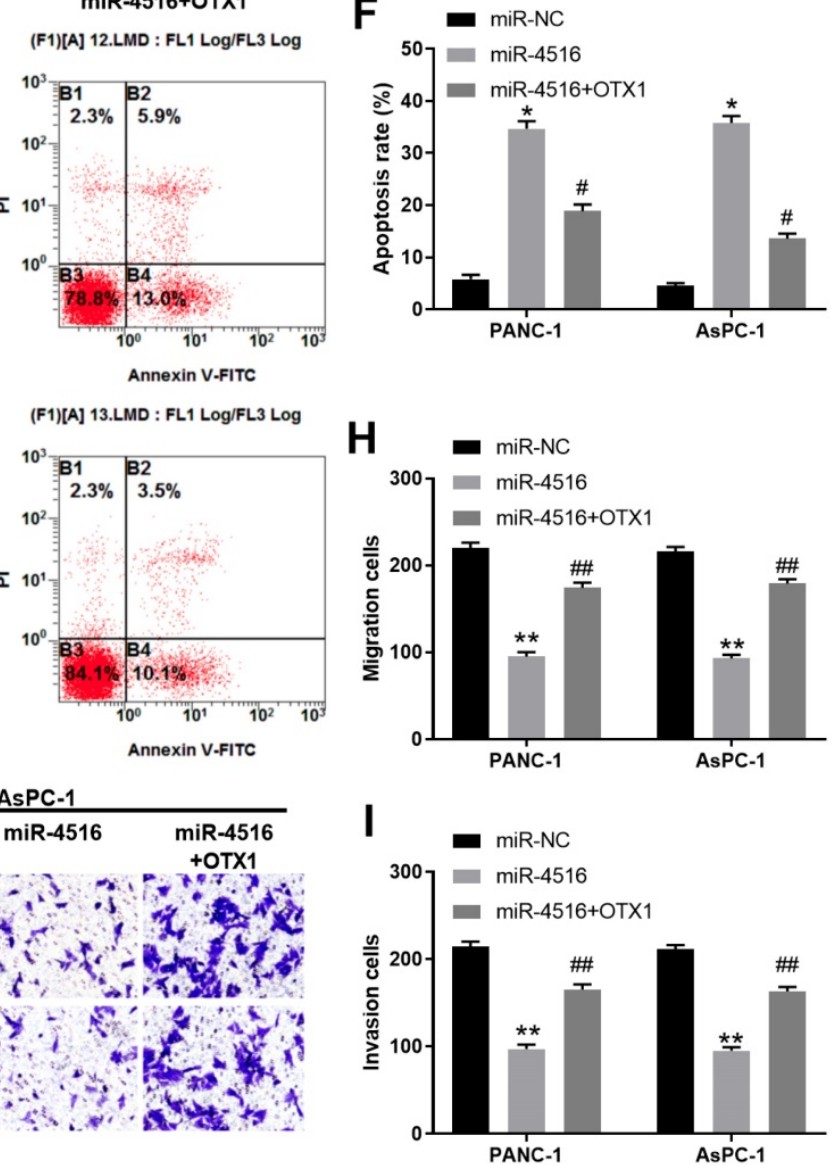

Figure 6. MiR-4516 inhibits pancreatic cell growth, migration and invasion and promotes cell apoptosis via targeting OTX1. PANC-1 or AsPC-1 cells were transfected with miR-NC, miR-4516 mimics, or miR-4516 mimics + OTX1 overexpression vector. (A) The protein expression of OTX1 in PANC-1 or AsPC-1 cells was analyzed by western blot. (B) Cell viability was analyzed by CCK-8 assay at indicated time points. (C, D) DNA synthesis was analyzed by EdU/DAPI double staining. (E, F) Cell apoptosis was analyzed by Annexin V/PI double staining. (G-I) Cell migration and invasion was analyzed by transwell assay. *, $P<0.05 ; * *, P<0.01$. 


\section{Discussion}

Mounting evidence has demonstrated that miRNAs play critical roles in tumorigenesis, function as tumor suppressors or oncogenes [8]. MiR-4516 is a miRNA first identified to downregulate STAT3/CDK6/UBE2N signaling axis in PUVA induced apoptosis in keratinocytes [23]. In our study, we found that miR-4516 was down-regulated in pancreatic cancer tissues and cell lines, indicating miR-4516 might be a tumor suppressor. We demonstrated that overexpression of miR-4516 inhibited pancreatic cancer cell proliferation, migration and invasion, while promoted cell apoptosis via negatively regulating OTX1. Furthermore, overexpression of miR-4516 suppressed tumor growth in vivo in pancreatic xenograft tumor model. These findings imply that miR-4516/OTX1 might be a useful therapeutic target in pancreatic cancer.

MiR-4516 was found highly expressed in dust-induced pulmonary fibrosis, which could be used as a potential biomarker for early diagnosis in patients with pneumoconiosis [24]. In hepatocellular carcinoma, lncRNA LSINCT5 acted as a competing endogenous RNA and sponged miR-4516 in regulated HMGA2 expression [25]. Interestingly, while our findings demonstrated that miR-4516 functioned as a tumor suppressor and inhibited pancreatic cancer development, miR-4516 is highly expressed in glioma patient samples and cell lines. Overexpression of miR-4516 promoted proliferation and invasion of glioblastoma cell via targeting PTPN14 [15]. The discrepancy of miR-4516 function in different tumors might due to the complicated tumor environment and regulatory network. A recent study revealed that miR-4516 overexpression suppressed the proliferation of breast cancer cells, especially triple negative breast cancer cells, indicating that miR-4516 might be used as an anti-cancer drug in breast cancer [26]. In this study, both in vitro and in vivo experiments suggest that miR-4516 suppresses pancreatic cancer development and progression.

Combined bioinformatics analysis predicts OTX1 is the downstream target of miR-4516 in pancreatic cancer. Previous studies have shown that OTX1 was frequently upregulated in multiple cancers, including breast cancer, gastric cancer and colorectal cancer [18, 20, 27]. Knockdown of OTX1 induced cell apoptosis, and attenuated cell migration and invasion of gastric cancer cells [20]. In colorectal cancer, lncRNA FEZF1-AS1 regulated OTX1 expression by sponging miR-30a-5p [27]. OTX1 was also reported to be negatively regulated by miR-3196 and lncRNA ADPGK-AS1 in breast cancer [27]. We demonstrated that miR-4516 specifically down-regulated OTX1 expression in pancreatic cell lines. Lucifease reporter assay confirmed the posttranscriptional regulation of OTX1 by miR-4516. Moreover, miR-4516 exerted its tumor suppressor function via regulating OTX1 and overexpression OTX1 abrogated the effect of miR-4516 overexpression. However, whether OTX1 was also regulated by other miRNAs or IncRNAs remains to be further studied. Studies have demonstrated that OTX1 was involved in regulating ERK/MAPK signaling in hepatocellular carcinoma and p53 signaling in breast cancer [18, 28]. The function mechanism of OTX1 in pancreatic cancer is not clear.

In conclusion, our findings suggest that miR-4516 acts as a tumor suppressor in pancreatic cancer. Overexpression of miR-4516 inhibits pancreatic cancer cell proliferation, migration, and invasion and promotes cell apoptosis via negatively regulating OTX1. Therefore, miR-4516/OTX1 might serve as a novel therapeutic target for miRNA-based therapy in pancreatic cancer.

\section{Acknowledgments}

The project was supported by Natural Science Foundation of Shaanxi Province (No: 2019JQ-969); Natural Science Foundation of Shaanxi Province (No: 2020JZ-39); Xi'an Jiaotong University Education Foundation, XJTUEF (No: xjj2018141); the Innovation Talent Promotion Project Foundation of Shaanxi Province (No: 2018KJXX-058); the Hospital Fund of the Second Affiliated Hospital of the Health Science Center, Xi'an Jiaotong University (No: RC(GG)201708); and the National Natural Science Foundation of China, NSFC (No: 81402583).

\section{Competing Interests}

The authors have declared that no competing interest exists.

\section{References}

1. Bray F, Ferlay J, Soerjomataram I, Siegel RL, Torre LA, Jemal A. Global cancer statistics 2018: GLOBOCAN estimates of incidence and mortality worldwide for 36 cancers in 185 countries. CA Cancer J Clin. 2018; 68: 394-424.

2. Capasso M, Franceschi M, Rodriguez-Castro KI, Crafa P, Cambie G, Miraglia $\mathrm{C}$, et al. Epidemiology and risk factors of pancreatic cancer. Acta Biomed. 2018; 89: 141-6.

3. Lambert A, Schwarz L, Borbath I, Henry A, Van Laethem JL, Malka D, et al. An update on treatment options for pancreatic adenocarcinoma. Ther Adv Med Oncol. 2019; 11: 1758835919875568.

4. Rawla P, Sunkara T, Gaduputi V. Epidemiology of Pancreatic Cancer: Global Trends, Etiology and Risk Factors. World J Oncol. 2019; 10: 10-27.

5. Zhao C, Gao F, Li Q, Liu Q, Lin X. The Distributional Characteristic and Growing Trend of Pancreatic Cancer in China. Pancreas. 2019; 48: 309-14.

6. Schober M, Javed MA, Beyer G, Le N, Vinci A, Sund M, et al. New Advances in the Treatment of Metastatic Pancreatic Cancer. Digestion. 2015; 92: 175-84.

7. Fan JQ, Wang MF, Chen HL, Shang D, Das JK, Song J. Current advances and outlooks in immunotherapy for pancreatic ductal adenocarcinoma. Mol Cancer. 2020; 19: 32

8. Tan W, Liu B, Qu S, Liang G, Luo W, Gong C. MicroRNAs and cancer: Key paradigms in molecular therapy. Oncol Lett. 2018; 15: 2735-42. 
9. Hanna J, Hossain GS, Kocerha J. The Potential for microRNA Therapeutics and Clinical Research. Front Genet. 2019; 10: 478.

10. Peng Y, Croce CM. The role of MicroRNAs in human cancer. Signal Transduct Target Ther. 2016; 1: 15004.

11. Xu K, Zhang L. Inhibition of TUG1/miRNA-299-3p Axis Represses Pancreatic Cancer Malignant Progression via Suppression of the Notch1 Pathway. Dig Dis Sci. 2019.

12. Niu $Y$, Jin $Y$, Deng SC, Deng SJ, Zhu S, Liu $Y$, et al. MiRNA-646-mediated reciprocal repression between HIF-1alpha and MIIP contributes to tumorigenesis of pancreatic cancer. Oncogene. 2018; 37: 1743-58.

13. Yu Z, Zhao S, Wang L, Wang J, Zhou J. miRNA-339-5p Plays an Important Role in Invasion and Migration of Pancreatic Cancer Cells. Med Sci Monit. 2019; 25: 7509-17.

14. Li X, Lv Y, Hao J, Sun H, Gao N, Zhang C, et al. Role of microRNA-4516 involved autophagy associated with exposure to fine particulate matter. Oncotarget. 2016; 7: 45385-97.

15. Cui T, Bell EH, McElroy J, Becker AP, Gulati PM, Geurts M, et al, miR-4516 predicts poor prognosis and functions as a novel oncogene via targeting PTPN14 in human glioblastoma. Oncogene. 2019; 38: 2923-36.

16. Larsen KB, Lutterodt MC, Mollgard K, Moller M. Expression of the homeobox genes OTX2 and OTX1 in the early developing human brain. J Histochem Cytochem. 2010; 58: 669-78.

17. Sakurai Y, Kurokawa D, Kiyonari H, Kajikawa E, Suda Y, Aizawa S. Otx2 and Otx1 protect diencephalon and mesencephalon from caudalization into metencephalon during early brain regionalization. Dev Biol. 2010; 347: $392-403$.

18. Terrinoni A, Pagani IS, Zucchi I, Chiaravalli AM, Serra V, Rovera F, et al. OTX1 expression in breast cancer is regulated by p53. Oncogene. 2011; 30: 3096-103.

19. Yu K, Cai XY, Li Q, Yang ZB, Xiong W, Shen T, et al. OTX1 promotes colorectal cancer progression through epithelial-mesenchymal transition. Biochem Biophys Res Commun. 2014; 444: 1-5.

20. Qin SC, Zhao Z, Sheng JX, Xu XH, Yao J, Lu JJ, et al. Dowregulation of OTX1 attenuates gastric cancer cell proliferation, migration and invasion. Oncol Rep. 2018; 40: 1907-16.

21. Shi Z, Johnson JJ, Stack MS. Fluorescence In Situ Hybridization for MicroRNA Detection in Archived Oral Cancer Tissues. J Oncol. 2012; 2012: 903581.

22. Wei $\mathrm{H}$, Wei $\mathrm{H}$, Wang $\mathrm{H}$, Tian $\mathrm{Z}$, Sun $\mathrm{R}$. Activation of natural killer cells inhibits liver regeneration in toxin-induced liver injury model in mice via a tumor necrosis factor-alpha-dependent mechanism. Am J Physiol Gastrointest Liver Physiol. 2010; 299: G275-82.

23. Chowdhari S, Saini N. hsa-miR-4516 mediated downregulation of STAT3/CDK6/UBE2N plays a role in PUVA induced apoptosis in keratinocytes. J Cell Physiol. 2014; 229: 1630-8.

24. Huang R, Yu T, Li Y, Hu J. Upregulated has-miR-4516 as a potential biomarker for early diagnosis of dust-induced pulmonary fibrosis in patients with pneumoconiosis. Toxicol Res (Camb). 2018; 7: 415-22.

25. $\mathrm{Li} \mathrm{O}, \mathrm{Li} Z$ Tang $\mathrm{O}$ Li $\mathrm{Y}$, Yuan $\mathrm{S}$, Shen $\mathrm{Y}$, et al. Long Stress Induced Non-Coding Transcripts 5 (LSINCT5) Promotes Hepatocellular Carcinoma Progression Through Interaction with High-Mobility Group AT-hook 2 and MiR-4516. Med Sci Monit. 2018; 24: 8510-23.

26. Kim JE, Kim BG, Jang Y, Kang S, Lee JH, Cho NH. The stromal loss of miR-4516 promotes the FOSL1-dependent proliferation and malignancy of triple negative breast cancer. Cancer Lett. 2020; 469: 256-65.

27. Li J, Zhao LM, Zhang C, Li M, Gao B, Hu XH, et al. The IncRNA FEZF1-AS1 Promotes the Progression of Colorectal Cancer Through Regulating OTX1 and Targeting miR-30a-5p. Oncol Res. 2020; 28: 51-63.

28. Li H, Miao Q, Xu CW, Huang JH, Zhou YF, Wu MJ. OTX1 Contributes to Hepatocellular Carcinoma Progression by Regulation of ERK/MAPK Pathway. J Korean Med Sci. 2016; 31: 1215-23. 